\title{
SRL-ESA-TextSum: A Text Summarization Approach Based on Semantic Role Labeling and Explicit Semantic Analysis ${ }^{+}$
}

\author{
Muhidin Mohamed ${ }^{\mathrm{a}, *}$, Mourad Oussalah $^{\mathrm{b}}$ \\ ${ }^{a}$ Computer Science, School of Engineering and Applied Sciences \\ Aston Universty, Aston Triangle, Birmingham B4 7ET, UK \\ ${ }^{b}$ Centre for Ubiquitous Computing, Faculty of Information Technology \\ Computer Science, University of Oulu, P.O. Box 4500, 90014 Finland
}

\begin{abstract}
Automatic text summarization attempts to provide an effective solution to today's unprecedented growth of textual data. This paper proposes an innovative graph-based text summarization framework for generic single and multi document summarization. The summarizer benefits from two well-established text semantic representation techniques; Semantic Role Labelling (SRL) and Explicit Semantic Analysis (ESA) as well as the constantly evolving collective human knowledge in Wikipedia. The SRL and ESA methods are used to achieve sentence semantic parsing and to represent its words as a vector of weighted Wikipedia concepts, respectively. The essence of the developed framework is to construct a unique concept graph representation underpinned by semantic role-based multi-node (under sentence level) vertices for summarization. We have empirically evaluated the summarization system using the standard publicly available dataset from Document Understanding Conference 2002 (DUC2002). Experimental results indicate that the proposed summarizer outperforms all state-of-the-art related comparators in the single document summarization based on the ROUGE-1 and ROUGE-2 measures, while also ranking second in the ROUGE- 1 and ROUGE-SU4 scores for the multi-document summarization. Results also tell that the system is scalable, i.e., varying the evaluation data size is shown to have little impact on the summarizer performance, particularly for the single document summarization task. In a nutshell, the findings demonstrate the power of the role-based and vectoral semantic representation when combined with the crowd-sourced knowledge base in Wikipedia.
\end{abstract}

Keywords: Text summarization, Semantic role labeling, Wikipedia concepts, Concept graphs, Semantic similarity, Iterative ranking algorithm.

\section{Introduction}

Text Summarization (TS) is a Natural Language Processing (NLP) task aimed to reduce original text documents to a short substitute summary which retains the most important facts of the source document. Summarization can be achieved by either extracting a group of sentences from an original source document and concatenating them called extraction,

\footnotetext{
${ }^{*}$ Corresponding author

Email address: mam256@alumni.bham.ac.uk (Muhidin Mohamed)

${ }^{+}$Most of this work was done while a PhD student at the Uiversity of Birmingahm.
} 
or generating a novel summary text representing its main gist referred to as abstraction. From an input perspective, a summary can either be sourced from one document through a process called single-document summarization, or from a collection of related documents which is known as multi-document summarization. Depending on the desired content, a summary is either a query-focused (tailored to a user query), or a topic-focused (containing document gist).

Today's increasing number of news sites, sent emails, customer product/service/travel reviews, user-generated social media content and QA communities all contribute to the rapid growth of already accelerating volume of textual information. The amount of information indexed in the Internet is estimated to be over 4.5 billion pages ${ }^{2}$ However, as the volume of generated structured and unstructured text increases exponentially, it renders the task of developing effective text summarization systems rather appealing. Therefore, advancing the research on TS is most needed than ever before due to the overwhelming growth of the Internet texts [1. Alongside this, the availability of full-fledged lexical knowledge sources and the powerful text semantic analysis tools inspire an extensive exploration of semantic $\&$ knowledge based summarization methods. This is also motivated by the assertion that using semantic knowledge holds the potential for further improvements in text summarization research and therefore needs more research investigation [2].

In this paper, we investigate a graph-based summarization approach using semantic role labelling for sentence level semantic parsing and Wikipedia as an external knowledge source. The SRL is a semantic parsing technique in NLP which identifies the semantic arguments associated with the predicate verbs of a sentence. It classifies the semantic roles of syntactic arguments within a given frame of the sentence and with respect to the predicate. The use of the proposed SRL-ESA Wikipedia graph-based generic summarization model is motivated by the following: the successful application of Wikipedia-based metric to the tasks of named entity semantic relatedness and query-focused summarization in our previous work [3, 4, the high lexical coverage of Wikipedia which led to its popularity as a reliable lexical resource for different NLP tasks such as semantic representation [6], word semantic similarity [5] and text classification [7]; the status quo in which current knowledge-based summarization methods underestimate the importance of sentence syntactic order and semantic roles, consequently undermining an accurate similarity computation and hence leading to poor scoring functions for summary extraction; the computation of word similarities in isolation from the surrounding context, thus ignoring significant semantic information conveyed by these words if associated with their roles when analysing them semantically. The chief contributions of this paper are:

1. First, we used semantic role labelling to build a semantic representation of documents and then paired matching semantic roles for any two compared sentences before mapping them onto their corresponding concepts in Wikipedia.

2. Next, we proposed a weighted semantic graph where each sentence is modeled as a multi-node vertex containing the Wikipedia concepts of its semantic arguments.

3. Finally, we implemented a single \& multi-document summariser using the above mentioned semantic graph representation and empirically evaluated its performance using the standard DUC2002 dataset.

\footnotetext{
${ }^{2}$ http://www.worldwidewebsize.com/
} 
The rest of the paper is organized as follows. Sections 2 and 3, respectively, cover the research objective and related works. Section 4 gives a brief introduction to the applied semantic analysis methods whereas Section 5 details the proposed summarization approach. Next, experiments for evaluating the system are presented in Section 6 before drawing paper conclusions in Section 7.

\section{Research Objective}

In this study, we combine four important components to model an extractive text summarizer; text semantic representation, effective concept-based sentence similarity measure, semantic role-based multi-node concept graph representation, and sentence ranking algorithm. The goal is to investigate the aggregate effect of the aforementioned 4 factors on the performance of topic-focused single and multi-document summarization. Our rationale behind this is to integrate the advantages of well-established text semantic analysis and representation techniques and the vast human knowledge encoded in the Wikipedia database.

The use of semantic parsing and word semantic roles is to address the problem of greedy word pairing approaches when computing sentence similarity. To achieve that semantic argument terms sitting the same role are grouped and mapped onto a vector of Wikipedia concepts. Wikipedia-mined concept vectors representing semantic arguments are then used to form sub-nodes of each sentence vertex in the document concept graph. We provide a detailed technical description of the proposed summarization system throughout the article and illustrate its functionality through a working example. We also present experimental evaluation to highlight the effectiveness of the method.

\section{Related Works}

Scoring document contents (e.g., words, phrases, sentences) is the most common method used in automatic extractive text summarization. The majority of today's implemented extractive summarizers adopt sentence scoring or graph-based sentence ranking. Prevailing sentence scoring and selection techniques in text summarization include graph-based representation [52], text semantic analysis [3], semantic similarity [5], sentence clustering [53, fuzzy reasoning [44, 49, sentence regression [55] and differential evolution [46, 54]. Several related works conducted a comparative study on a range of sentence scoring methods by examining the performance of their combinations for text summarization [51, 52.

Two powerfull but often underused semantic analysis tools for text summarization are the SRL and ESA methods. A few related works have independently utilised SRL for text summarisation. This includes feature-based approaches in association with SRL, such as the work of Khan et al. 9 where they used predicate argument structures to represent source documents; the proposal of Suanmali et al. 33 where the researchers combined statistical and SRL-based features; and schemes based on semantic frame and argument frequencies to identify key sentences [10, 11]. Some other studies used SRL together with an iterative graph-based ranking algorithm for text summarization, e.g., the work of Canhasi and Kononenko [18] who introduced a multilayered document similarity graph linking sentence semantic frames. Likewise, Yan \& Wan 12 used SRL tuples as additional nodes in a multi-level graph representation and treated them as independent units for sentence ranking. Moreover, the application of ESA to text summarisation is still in its infancy. On this subject, Sankarasubramaniam et al. 25] suggested a Wikipedia-based text summarisation algorithm using a bipartite sentence concept graphs to rank document sentences according to their concepts. 
In a more feature-based fashion, Zhou et al. 13 applied ESA to query-focussed text summarisation and integrated an ESA-based technique and traditional sentence features to score document sentences using machine learning algorithms.

In addition, graph-based representations are some of the most prevalent text analysis methods and have shown their effectiveness for text summarization 14, 15, 18, 19, 20, 21, 22 , 23. The conventional way of graph-based summarization uses document sentences as vertices, known as sentence-based document graphs. Erkan and Radev [14 proposed one of the most popular sentence-based graph representations for summarisation. Their LexRank algorithm is based on the eigenvector centrality concept. Similarly, Mihalcea and Tarau [15] presented TextRank, another graph-based ranking method constructed using content overlap. Both LexRank and TextRank are derivatives of the seminal PageRank algorithm [16]. Some graph-based approaches have cross-linked different levels of text granularities particularly tailored for multi-document summarization [18, 19, 20. In this way, Canhasi and Kononenko 21 used three-layer graph representation consisting of terms, sentences, and document vertices, and linked them via term-sentence and sentence-document links on top of the conventional sentence similarity graphs. In addition, the works of Wei et al. [19] and Wan [20] considered the influence of global information from the document clusters on local sentence evaluation. Contrary to the traditional way of representing source text units, concept graphs have also been emerging as alternative graph representation of the source texts 24, 25, 28, 22. Of this, Azadani et al. 22, and Plaza et al. 24, have both adapted such a method by modelling a biomedical summarization algorithm on concept graphs. Also, Zhuge [26] proposed a multi-dimensional summarization methodology to summarize various objects (including texts, pictures and videos) from multiple dimensions. The effectiveness of this methodology has been evaluated for text summarization 27. Concept graph modelling proved some success particularly in domain-specific areas such as biomedical and news summarizations [28].

While the advantages of semantic parsing for text summarisation have been highlighted in previous semantic-based works [9, 10, 12, the uniqueness of our approach is that it combines the strengths of leveraging text semantic analysis and representation techniques with a high coverage crowd-sourced human knowledge. Also, the distinction between the current SRL-ESA Wikipeida based summarisation model and the preceding graph-based methods is the use of under-sentence semantic argument links in the construction of document concept graphs, which gives this approach an advantage over others. This is because, intuitively, pairing matching semantic roles captures more semantics than applying indiscriminate word pairing greedily. Thus, realizing the strengths of world knowledge and semantic analysis, our approach adapts both SRL and ESA techniques for extractive text summarisation underpinned with the encyclopedic knowledge in Wikipedia.

\section{Used Semantic Analysis Techniques}

\subsection{Semantic Role Labeling (SRL)}

SRL is a technique for sentence level semantic analysis. It segments the text and identifies the semantic role of each syntactic constituent word with reference to the main predicate verb in the sentence. On the other hand, sematic roles are the basic units of a semantic frame which is a collection of facts that specify "characteristic features, attributes, and functions of a denotatum, and its characteristic interactions with things necessarily or typically associated with it" 29. Relations between semantic frames and word meanings, as encoded in FrameNet lexical database [30, represent the core of Frame Semantics theory [31. PropBank, another 
relevant resource, houses a large corpus of human annotated predicate-argument relations added to the syntactic trees of the Penn Treebank 35. The basic concept of Frame Semantics is that word meanings must be described in relation to semantic frames.

Linked with the above, sentence semantic parsing is a fundamental task that has a large number of immediate NLP applications including plagiarism detection 34 and text summarization [33. With the help of human annotated resources such as ProbBank [32] and FrameNet [30, the development of automatic systems for the identification of semantic roles is a well investigated current research topic in NLP. One of the seminal works about building automatic semantic role labellers was proposed by Gildea and Jurafsky [35. Their system is based on statistical classifier trained on hand-annotated dataset from FrameNet. Recently, Collobert et al. 36 proposed a unified neural network architecture and learning algorithm which was applied to different natural language processing tasks such as part-of-speech tagging, chunking, named entity recognition, and semantic role labelling. Their algorithm learns internal data representations using vast amounts of mostly un-annotated training data. They have built freely available software called SENNA which we used for the prediction of semantic roles in our work. One of the attractive features of this tagging system is its good performance in terms of its speed and the minimal computational requirements.

In a nutshell, the primary goal of SRL is to single out all component words that fill semantic roles for a predicate verb and then assign them the corresponding semantic role tags. It is usually stated that SRL answers the question of basic event structures such as who did what to whom when where and why. Example 1 illustrates how SRL works.

Table 1: Semantic role arguments and associated labels

\begin{tabular}{llll}
\hline \multicolumn{2}{l}{ Core } & Arguments & \multicolumn{2}{l}{ Non-core } & Arguments \\
\hline Label & Modifier & Label & Modifier \\
\hline V & Verb & AM-DIR & Direction \\
A0 & Subject & AM-ADV & Adverb \\
A1 & Object & AM-LOC & Location \\
A2 & Indirect Object & AM-TMP & Temporal marker \\
A3 & Start Point & AM-MNR & Manner \\
A4 & End Point & AM-DIS & Discourse marker \\
A5 & Direction & AM-PRP & Purpose \\
- & - & AM-NEG & Negation \\
- & - & AM-EXT & Extent \\
- & - & AM-PNC & Proper noun \\
\hline
\end{tabular}

\section{Example 1:}

John finalized the experiment and reported the findings to the supervisor.

\begin{tabular}{|c|c|c|c|c|c|c|c|c|c|c|c|}
\hline & John & finalized & the & experiment & and & reported & the & findings & to & the & supervisor \\
\hline finalize. 01 & $\mathrm{~A} 0$ & & & A1 & & & & & & & \\
\hline report. 01 & A0 & & & & & & & A1 & & & A2 \\
\hline
\end{tabular}

Figure 1: Example 1 semantically parsed with SRL 
Figure 1 shows the sentence in Example 1 semantically parsed with the Lund Semantic Role Labeler ${ }^{3}$. The semantic parser recognises the predicate verbs and their associated arguments. Core SRL arguments include Agent (aka subject), Theme (aka direct object), Instrument, among others. They also include adjunctive arguments indicating Locative, Negation, Temporal, Purpose, Manner, Extent, Cause, etc. The list of semantic role arguments and associated tags are given in Table 1. Figure 1 indicates that the example sentence has two verbs: finalized and reported. The labels A0, A1 and A2 in the figure indicate the subject, object and indirect object of the respective verb, whilst role-sets of the predicate verbs finalized, and reported are listed in Table 2. The hyphen (-) in the table indicates that the predicate lacks this argument. One can note that the subject John is a common agent for both verbs.

Table 2: Verb-argument pairs for the example in Figure 1

\begin{tabular}{lcccc}
\hline \hline Aerbs & Arguments & A0 & A1 & A2 \\
\cline { 1 - 4 } $\begin{array}{l}\text { Finalize } \\
\text { Report }\end{array}$ & $\begin{array}{c}\text { John } \\
\text { John }\end{array}$ & $\begin{array}{c}\text { the experiment } \\
\text { the findings }\end{array}$ & to the supervisor \\
\hline
\end{tabular}

\subsection{Explicit Semantic Analysis (ESA)}

ESA is a Wikipedia-based technique for computing text semantic relatedness proposed by Gabrilovich and Markovitch 37. The ESA procedure maps text snippets to a vector space containing Wikipedia-derived concepts. The technique assumes that Wikipedia articles represent natural language concepts and, hence, mapping text fragments to their accommodating concepts is perceived as a representation of the text meaning. Formally speaking, ESA constructs an inverted index from the Wikipedia database and uses that to represent input texts by building ordered and weighted Wikipedia concepts. This is done by iterating over each token of a text to be interpreted. The actual computation of the text semantic relatedness is then performed by comparing translated vectors of two texts using cosine similarity. Figure 2 demonstrates the explicit semantic analysis process. For two natural language fragments to be compared the semantic interpreter iterates over each word of every text, retrieves its corresponding entry from the inverted index, and represents the word by the retrieved vector of concepts weighted by their TF-IDF scores.

More formally, if $T=\left\{w_{i}\right\}$ is the input text, $\overrightarrow{K_{w_{i}}}$ is the inverted index entry for word $w_{i}$, where $K_{w_{i}}$ represents the strength of association for $w_{i}$ with the Wikipedia concept set $C=\left\{c_{1}, c_{2}, \ldots, c_{N}\right\}$, then the semantic interpretation for $\mathrm{T}$ is the vector $V=\left\{v_{1}, v_{2}, \ldots, v_{N}\right\}$. Each element in $\mathrm{V}$ quantifies the association of the corresponding concept $c_{j}$ to the text $\mathrm{T}$, and is defined as $\sum_{w_{i} \in T} t f . i d f_{w_{i}} * k_{w_{i}}$. The TF-IDF (term frequency- inverse document frequency) is one of commonest weighting schemes in information retrieval [39]. It calculates the weight of a word as per expression (1).

$$
t f . i d f(w, d)=t f_{w},{ }_{d} \cdot \log \frac{N}{n_{w}}
$$

\footnotetext{
${ }^{3}$ http://barbar.cs.lth.se:8081/parse
} 


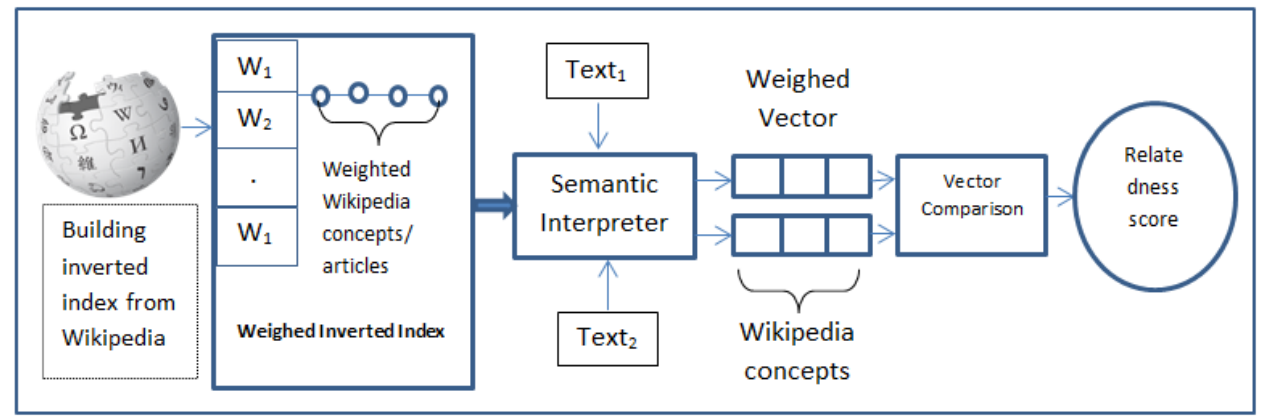

Figure 2: Explicit Semantic Analysis

Where $t f_{w, d}$ is the frequency of word $w$ in document (Wikipedia article) $d, n_{w}$ is the number of documents in which $w$ occurs, and $N$ is the number of documents in the text collection (size of English Wikipedia articles in our work). Once the text $T$ is mapped onto its corresponding Wikipedia concepts vector, the final stage of the ESA process is to compute the semantic relatedness. In other words, if $T_{1}$ and $T_{2}$ are two text fragments, their semantic relatedness, $\operatorname{SemRel}\left(T_{1}, T_{2}\right)$, is computed by comparing their respective vectors; $V_{1}$ and $V_{2}$ as in expression (2).

$$
\operatorname{SemRel}\left(T_{1}, T_{2}\right)=\frac{V_{1} \cdot V_{2}}{\left\|V_{1}\right\|\left\|V_{2}\right\|}
$$

ESA has been used for various NLP tasks such as text categorisation 40] and information retrieval [41].

\section{Proposed SRL-ESA Based Text Summarization Model}

To our knowledge, this work is the first study that combines Semantic Role Labeling and Wikipedia-based explicit semantic analysis for text summarisation. It improves the construction of document similarity graphs for graph-based text summarisation. Figure 3 shows a block diagram of the the proposed text summarization model. The process involves two principle stages. In the first stage, we perform two parallel processing tasks; the experimental data pre-processing followed by semantic parsing with SRL and argument grouping on the one hand, and constructing an inverted index database of Wikipedia concepts on the other hand. In addition, summarizing multi-documents involves additional step in which documents of each cluster are merged to form a single cluster document, as described in Section 5.1. In the pre-processing step, we processed the experimental dataset by converting the raw document texts to semantic linguistic units using some basic NLP tasks including document segmentation, sentence tokenization, part-of-speech tagging, word stemming and the removal of stop words. Document texts are parsed with semantic role labeling to identify the semantic frames and associated arguments. This is followed by semantic argument grouping in which all argument terms of similar semantic roles are collected and linked to their modifiers. The proposal makes use of Wikipedia database dump to construct an inverted index file (top right of Figure 3). The inverted index file is created with the aid of Appache Lucene Library 4 to provide a mapping of argument terms to accommodating Wikipedia

\footnotetext{
${ }^{4}$ http://lucene.apache.org.
} 


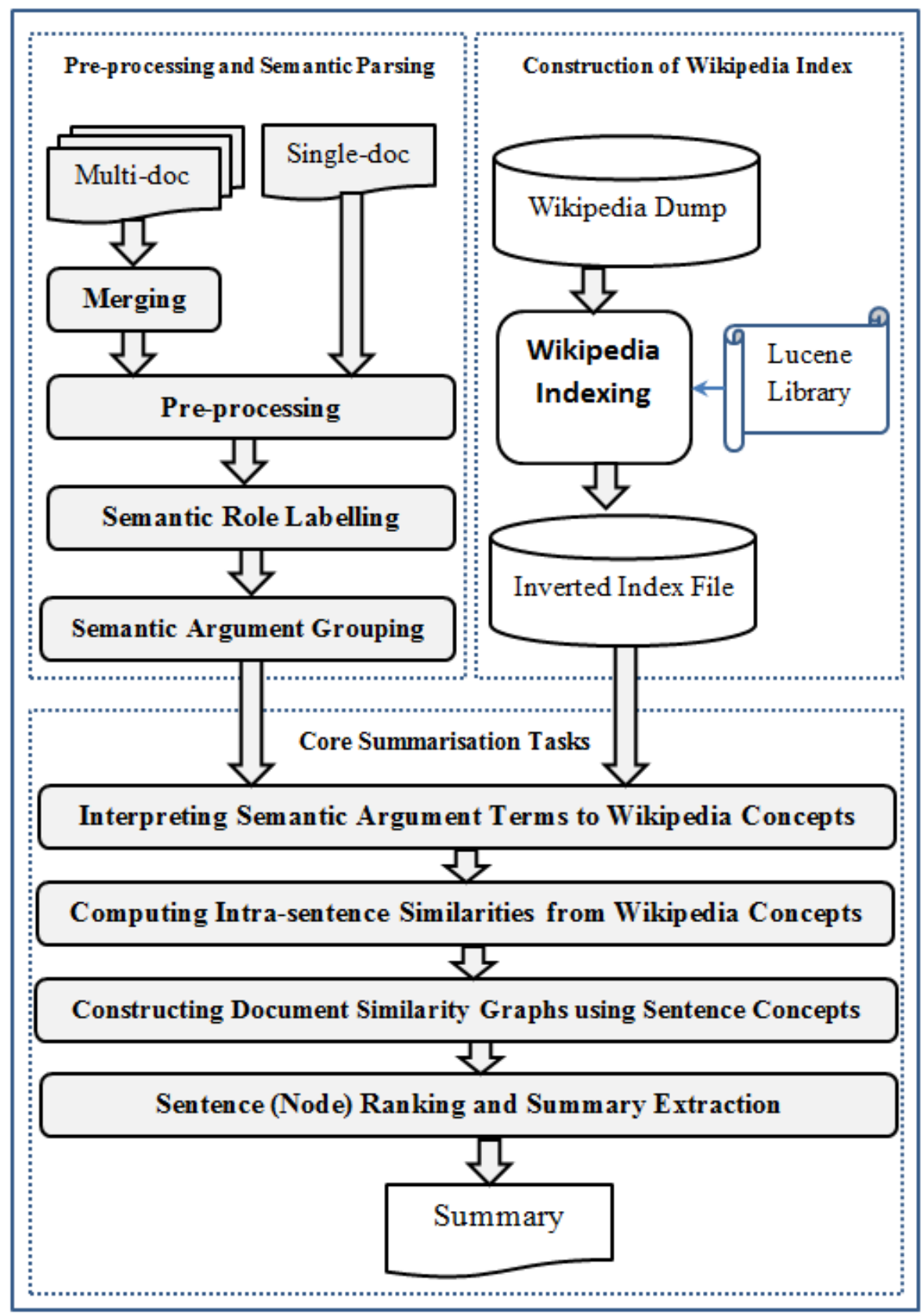

Figure 3: SRL-ESA graph-based text summarization system

concepts using the ESA approach 37] explained in Section 4.2. The next stage deals with core summarization tasks, as will be detailed in the following sections. 


\subsection{Merging Multi-document Clusters}

In multi-document summarization, a single summary is sought from across many documents that describe the same topic. These documents, which are written by different authors, are normally taken from different news sources. Unlike single document summarisation, the process of summarising a collection of related documents poses a number of other challenges including a high degree of redundancy, which conceivably results from merging multiple descriptions of the same topic, and the ordering of the extracted summary sentences. To reduce redundancy, different summarization approaches used different methods such as measuring sentence similarity [54, 58, using the seminal MMR algorithm and its derivative [1, 48, 56, and exploiting clustering algorithms [57. Besides, sentence ordering remains a less studied problem in MDS. On this subject, Bollegala [59] combined four criteria (chronology, topicalcloseness, precedence, and succession) to develop a sentence ordering approach for multi document summarization. In this work, we have designed a pre-processing stage to mitigate these challenges. Firstly, related documents of each cluster to be summarised are merged together to form a single cluster document while arranging the entire text in the order of the source documents' timeline. We then iteratively removed similar sentences to exclude repeated content. This is done by finding the similarity of each sentence with the rest of the cluster sentences and removing those with high similarity scores. This produces a unified cluster document with minimized information repetition.

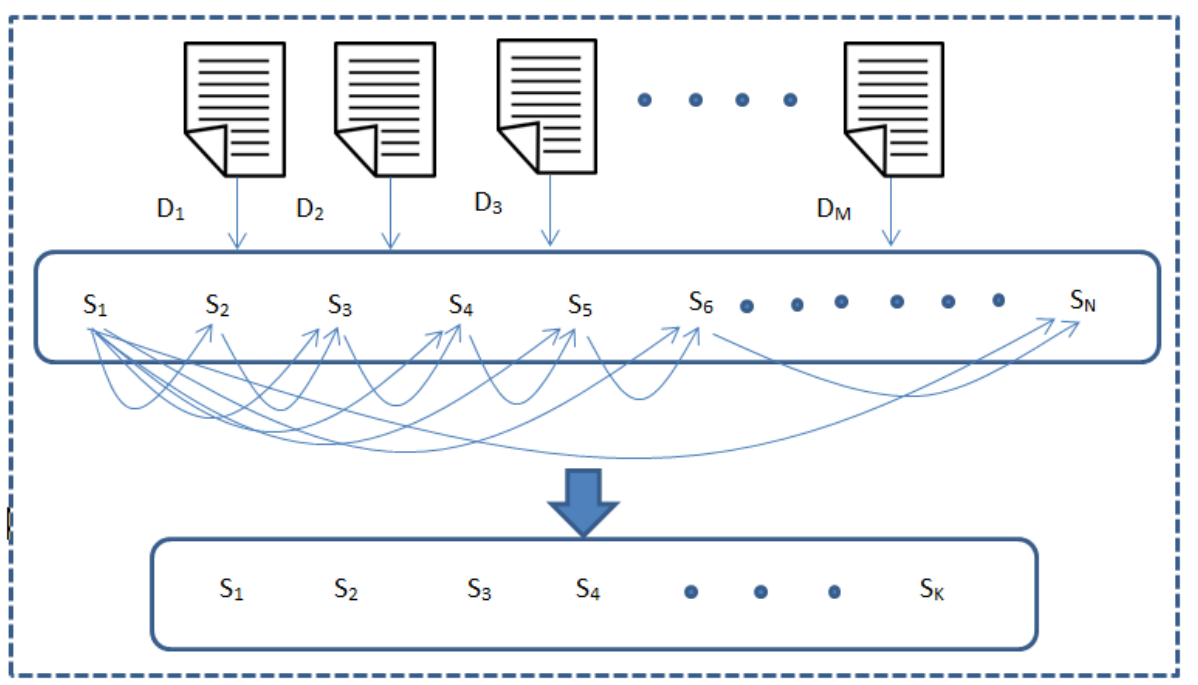

Figure 4: Merging cluster documents with redundancy removal

More formally, if $C=\left\{D_{1}, D_{2}, D_{3}, \ldots, D_{M}\right\}$ is a cluster of $M$ documents to be summarised, we combine all sentences of the document collection to obtain a flattened cluster, $C=\left\{S_{1}, S_{2}, S_{3}, S_{4}, S_{4}, S_{4}, \ldots, S_{N}\right\}$, where $N$ is the total number of cluster sentences. Next, a filtering process is applied to $C$ in order to sieve cluster sentences by discarding all highly similar sentences to the current one. Figure 4 describes the cluster merging process. For better readability, the figure indicates outward arrows for $S_{1}$ only, but the same logic applies to the rest of the sentences. By this merging, we remove $(N-K)$ sentences where $N \geq K$. 


\subsection{Computing SRL-ESA Based Semantic Similarity}

The fundamental building block of our summarization system is the determination of the role-based semantic similarity for intra-sentence similarity graphs and redundancy avoidance. Developing more effective sentence similarity measures are now believed to hold the future potential of extractive text summarization [38. To calculate the semantic similarity, we first pre-processed documents by merging each collection of related documents (multi-document summarisation only) and then segmented both single and multi-documents into sentences. Next, we constructed the semantic representation of each sentence by parsing it with semantic role labelling software. This semantic parsing aims at discovering semantic frames and associated arguments for each document sentence. The semantically parsed sentences are then formatted to a custom template for subsequent processing.

For exemplification, consider Example 2 of highly semantically related sentences.

\section{Example 2:}

$S_{1}$ : FIFA was accused of corruption.

$S_{2}$ : FIFA was officially investigated for corruption.

Applying semantic parsing identifies the predicate verbs of each sentence. In this case, each sentence has a single predicate verb, accuse for sentence 1 and investigate for sentence 2, and hence one primary semantic frame each. The role set of each predicate is classified according to the semantic roles they sit with respect to the verb. With this respect, three arguments, namely, A1 (direct object), A2 (indirect object) and AM-MNR (manner) are identified in both sentences. Table 3 shows a breakdown of both sentences in Example 2 into semantic frames indicating the semantic role that each token fills in the predicate.

\begin{tabular}{lll|lll}
\hline \multicolumn{6}{l}{ Table 3 } \\
\hline \multicolumn{5}{l}{$S_{1}$ predicates and sed Example 2 sentences with } & \multicolumn{4}{l}{ their predicates and semantic role tags } \\
\hline Terms & Predicates & Role Tags & Terms & Predicates & Role Tags \\
\hline FIFA & - & A1 & FIFA & - & A1 \\
was & - & 0 & was & - & 0 \\
accused & accused & V & officially & - & AM-MNR \\
of & - & B-A2 & investigated & investigated & S-V \\
corruption & - & E-A2 & for & - & B-A2 \\
& & & corruption & - & E-A2 \\
\hline
\end{tabular}

Formally, let $S_{1}$ and $S_{2}$ be two sentences consisting of semantic frames $f_{1}$ and $f_{2}$ respectively. Let $R_{1}=\left\{r_{1}, r_{2}, \ldots, r_{k}\right\}$ and $R_{2}=\left\{r_{1}, r_{2}, \ldots, r_{l}\right\}$ be the semantic role sets associated with $f_{1}$ and $f_{2}$ where $k$, and $l$ are the numbers of arguments in the semantic frames. From the two role sets of the semantic frames, we select the common roles, $R_{c}=r_{1}, r_{2}, \ldots, r_{m}$, co-occurring in both sentences. All other unshared semantic roles are discarded from the calculation of the semantic similarity. This is because of the intuition that an accurate similarity can be captured by comparing the semantic arguments corresponding to matching semantic roles. Having identified all shared semantic roles, the next step of our similarity computation involves building a Role-Terms Table for each sentence. The Role-Terms Table is a table that lists all shared semantic roles along with their related term vectors. For instance, if we assume that $T V=\left\{W V_{1 i}, W V_{2 i}, \ldots, W V_{m i}\right\}$ are term vectors related to the semantic roles $r_{1}, r_{2}, \ldots, r_{m}$ of sentence $i$, the Role-Terms Table for Example 2 can be 
constructed as in Table 4 , a better organization of the data in Table 3 . The table shows argument terms of the shared roles for the example sentences after normalizing tokens, removing the noise (stop) words, and leaving semantic content words. Since there are few words in the example pair, we created a single Role-Terms Table for both sentences.

\begin{tabular}{|c|c|c|}
\hline Role (Arg.) label & $S_{1}$ argument terms $\left(W V_{i 1}\right)$ & $S_{2}$ argument terms $\left(W V_{i 2}\right)$ \\
\hline $\mathrm{V}$ & Accuse & Investigate \\
\hline A1 & FIFA & FIFA \\
\hline $\mathrm{A} 2$ & corruption & corruption \\
\hline
\end{tabular}

Once Role-Terms are constructed, the next step of our SRL-ESA based semantic similarity calculation is to translate the argument terms to their corresponding Wikipedia concepts. This is aided by a pre-built inverted index file containing a mapping of English content words to a weighted vector of hosting Wikipedia concepts as described in the preceding section. Continuing from our previous discussion, we interpret the Role-Terms Table to a table of concept vectors where each concept vector replaces argument terms filling the same semantic role. If $W V_{i j}$ represents the argument term(s) of role $i$ from sentence $j$, it translates to $C V_{i j}$, the weighed vector of Wikipedia concepts corresponding to $W V_{i j}$. For illustration purpose, Table 5 shows the first 5 Wikipedia concepts corresponding to the argument term corruption along with their unique Wikipedia ID numbers and TF-IDF weights.

Table 5: Top 5 Wikipedia concepts of the argument term: Corruption

\begin{tabular}{lll}
\hline Wikipedia ID\# & Concepts & TF*IDF weight \\
\hline 20055663 & Prevention of Corruption Act & 0.5399063230 \\
2110801 & Corruption (linguistics) & 0.5140590668 \\
25239439 & Corruption in the United States & 0.4959531128 \\
3174020 & Corruption Perceptions Index & 0.45036080479 \\
66241 & Transparency International & 0.4280707538 \\
\hline
\end{tabular}

Table 5 demonstrates the interpretation of the argument terms to corresponding Wikipedia concept vectors. Next, the actual semantic similarity between the two sentences is computed using these representative natural concepts. If $r_{1}, \ldots, r_{m}$ denote the shared semantic roles between two sentences where $m$ is the number of the common roles, we use the Wikipedia concept vectors translated from the argument terms filling in these semantic roles. More formally, let $\left\{C V_{1 k}, \ldots, C V_{i k}\right\}$ and $\left\{C V_{1 l}, \ldots, C V_{i l}\right\}$ be the concept vectors interpreted from the argument terms of the common roles between sentences $k$ and $l$. The semantic similarity between sentences $\mathrm{k}$ and $\mathrm{l}$ is calculated as the average role similarities (RSim) obtained from the corresponding shared role sets. This is defined in Equation (3) where $i$ denotes the shared roles.

$$
\operatorname{Sim}_{s r l-e s a}\left(S_{k}, S_{l}\right)=\frac{1}{m} \sum_{i=1}^{m} R \operatorname{Sim}\left(C V_{k i}, C V_{l i}\right)
$$

The $R \operatorname{Sim}\left(C V_{k i}, C V_{l i}\right)$ is computed using individual concepts representing the original argument terms as formulated in (4). In Equation (4), $w c_{j k}$ represents the $t f-i d f$ weight of term $j$ with respect to its corresponding concept from argument role $i$ of sentence $k$ while 
$w c_{j l}$ is the $t f-i d f$ weight of term $j$ with respect to its corresponding concept from argument role $i$ of sentence $l$.

$$
R \operatorname{Sim}\left(C V_{k i}, C V_{l i}\right)=\frac{\sum_{j=1} w c_{j k} * w c_{j l}}{\sqrt{\sum_{j=1} w c_{j k}^{2}} \sqrt{\sum_{j=1} w c_{j l}^{2}}}
$$

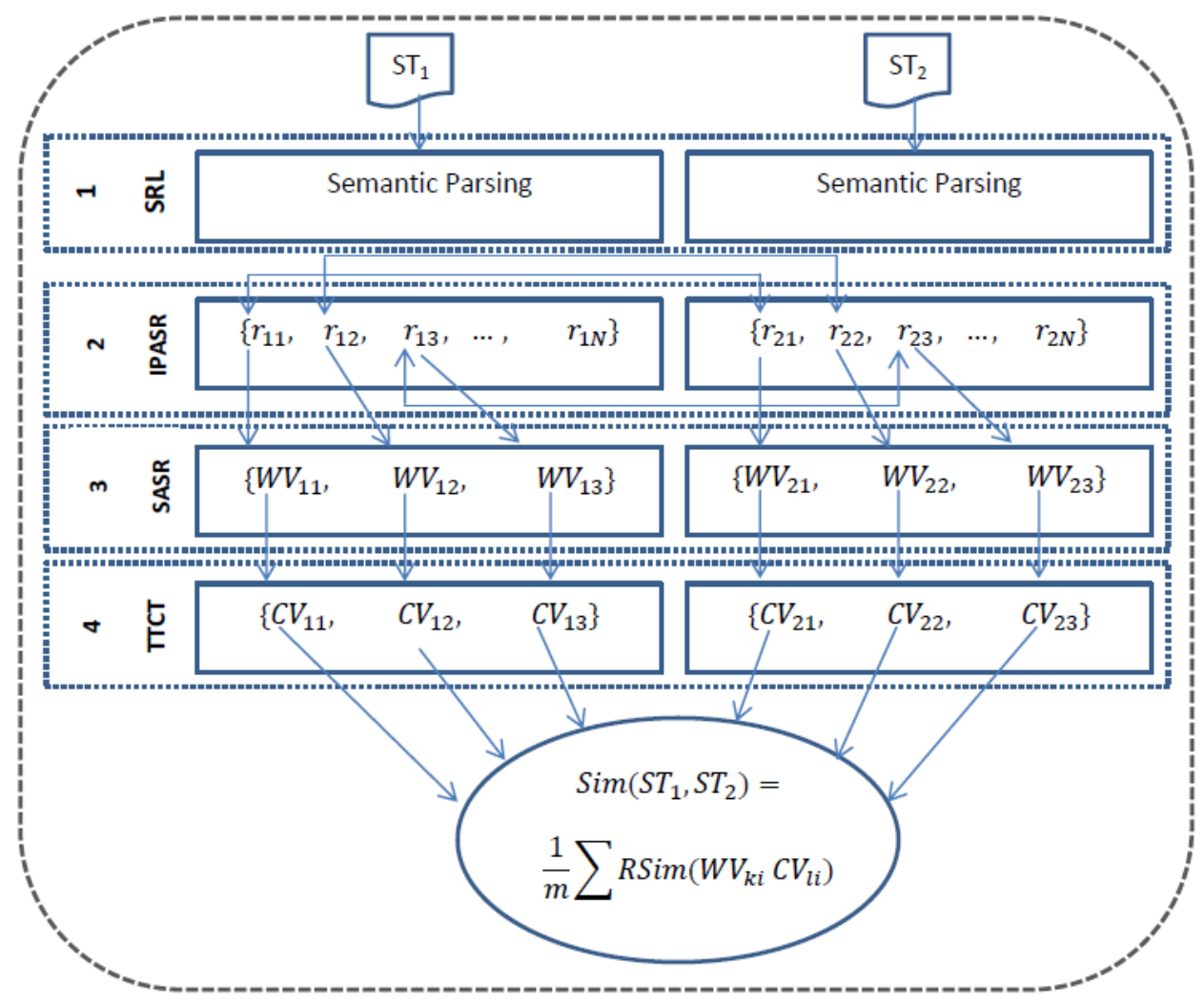

Figure 5: SRL-ESA based semantic similarity computation for short texts

Figure 5 summarizes the SRL-ESA based procedure for calculating the semantic similarity between two short texts $S T_{1}$ and $S T_{2}$. The figure demonstrates four procedural stages with the assumption of three shared roles.

1. The first step applies the semantic parsing by using semantic role labelling (SRL). The input to this stage is short texts and the output is semantically tagged/parsed texts.

2. Secondly, the predicate verbs for the texts are detected together with their semantic role sets. Therefore, this stage is called Identification of Predicates and Associated Semantic Roles, shortly abbreviated as IPASR.

3. Our process recognises that all semantic roles are not shared in typical short texts and selects the arguments of common semantic roles in the third stage. This is referred to as Selecting Arguments of Shared Roles (SASR). 
4. The final stage translates all grouped argument terms to their corresponding weighted Wikipedia concepts before carrying out the actual similarity calculation. This stage is known as Terms to Concepts Translation, or TTCT.

\subsection{Semantic Graph Representation of Documents}

In the next step of the proposed summarization approach, every document is represented as a weighted undirected graph where the sentence concepts form the nodes (vertices) and their semantic similarities weight the edges. More formally, let $G=(V, E, \alpha, \beta)$ be a weighed undirected graph with the set of vertices $\mathrm{V}$ representing sentence concept vectors and the set of edges $(E \subseteq V)$ linking the vertices. The parameters: $\alpha: V \rightarrow \mathbb{R}_{+}$and $\beta: E \rightarrow \mathbb{R}_{+}$ are functions defining the vertices rankings and edge weights respectively. In addition to the sentence-based graph representation, we used semantic links under sentence level. In other words, each sentence is modeled as a multi-node vertex using the Wikipedia concept vectors $\left(C V_{i}\right)$ of the semantic argument terms. Figure 6 shows the semantic argument representation (A) and the sentence level similarity graphs used for similarity computation and sentence ranking, respectively. The graph vertices are ranked using PageRank algorithm [16]. The edge-weights are formulated as per Equation 5. For single document summarization, the weights are measured in a slightly different way than for multi-document summarization. For the former, the similarity between each sentence with the document title is considered in addition to the intra-sentence similarities. This is becuase, unlike multi-documents, each single document in the dataset has a unique title. Intuitively, having a high semantic similarity with the document title indicates an additional importance of that given sentence in the document (see the document example in Figure 7 and Tables (7-80).

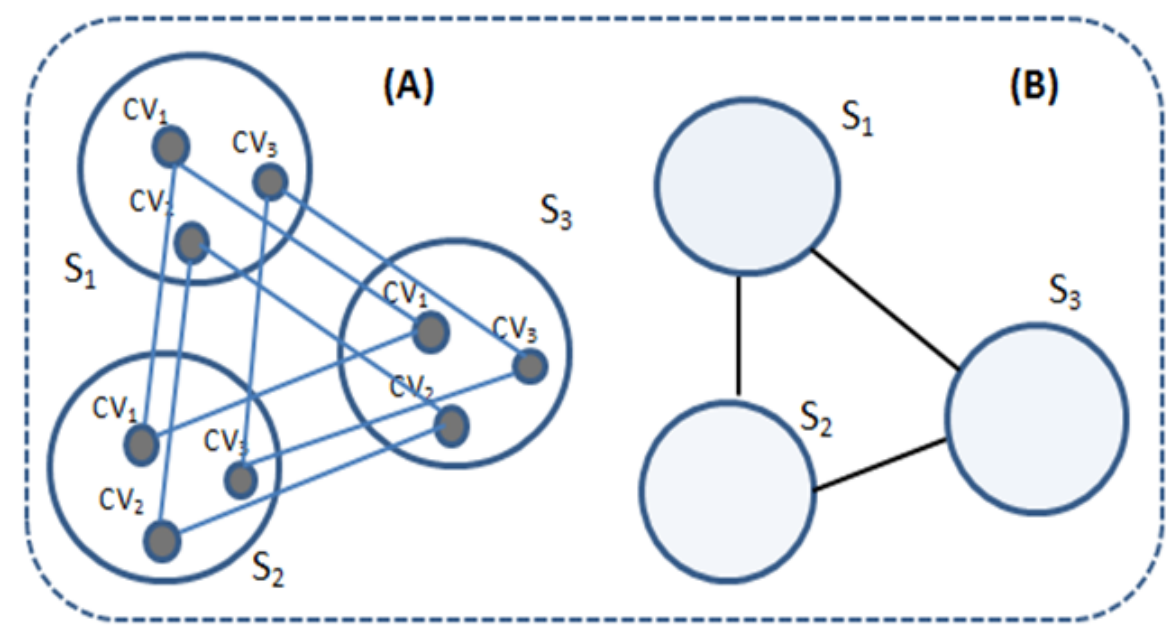

Figure 6: Semantic argument level (A) and sentence level (B) document similarity graphs

$$
\text { EdgeWeight }(\beta)= \begin{cases}\operatorname{Sim}_{\text {srl-esa }}\left(S_{1}, S_{2}\right)+T \operatorname{Sim}\left(S_{1}, S_{2}, T\right) & \text { for } S D S \\ \operatorname{Sim}_{\text {srl-esa }}\left(S_{1}, S_{2}\right) & \text { forMDS }\end{cases}
$$

where $S_{i}$ denotes sentence $i, T$ is the document title, $S D S$ (resp. $M D S$ ) represents single document summarization (resp. multi-document summarization), $\operatorname{Sim}_{\text {srl-esa }}\left(S_{1}, S_{2}\right)$ is 
the SRL-ESA based similarity measure formulated in (3) and $T \operatorname{Sim}\left(S_{1}, S_{2}, T\right)$ is the tile similarity which is given in (6).

$$
\operatorname{TSim}\left(S_{1}, S_{2}, T\right)=0.5 *\left(\operatorname{Sim}_{\text {esa }}\left(S_{1}, T\right)+\operatorname{Sim}_{\text {esa }}\left(S_{2}, T\right)\right)
$$

The title-sentence similarity is calculated using Wikipedia concepts without semantic parsing due to the nature of most document titles which lack predicates and semantic frames. It is also worth noting that, in some rare cases, the sentences without predicate verbs are not included in the graph representation. This is because the SRL based semantic analysis cannot be applied to such sentences lacking semantic frames.

Document ID: AP000825-0099, Cluster ID: d070f, Dataset: DUC2002

Title: Report - Honecker Unlikely To Go to Trial in East Germany

$S_{1}$ : Ousted East German leader Erich Honecker will not stand trial in East Germany as long as the formerly Communist country exists, a West German newspaper reported.

$S_{2}$ : The Hamburg-based Bild am Sonntag said Saturday that it would report in its Sunday editions that Honecker could be prosecuted in a united Germany, however, for violation of property laws.

$S_{3}$ : Bild quoted Guenter Seidel, an East German prosecutor, as saying that Honecker had used 42 million for stocking a private housing estate for leaders of the former Communist government.

$S_{4}$ : However, Seidel said that the investigation was not far enough along to determine whether charges could be filed against Honecker before East Germany merges with West Germany on Oct.

$S_{5}$ : Negotiators are still working out the merger of the two German legal systems.

$S_{6}$ : Honecker, 78, was ousted as East Germany's leader on Oct. 18, paving the way for the country's first freely elected government in March.

$S_{7}$ : Honecker is in poor health and remains confined to a Soviet military hospital in Beelitz outside East Berlin.

$S_{8}$ : He is under investigation on allegations of abuse of power, corruption, harboring terrorists and issuing shoot-to-kill orders to prevent East Germans from escaping to West Germany when he served as the country's leader.

$S_{9}$ : Bild said that Erich Mielke, the ex-head of East Germany's former secret police, was also unlikely to go to court in East Germany.

$S_{10}$ : I am at the end. I am a dead man, Bild quoted Mielke, 82, as saying at his last interrogation.

Figure 7: An example document to be summarized

\subsection{Iterative Sentence Ranking and Summary Extraction}

We applied PageRank algorithm to the document similarity graphs to rank and identify the most important sentences to be extracted as a summary. The PageRank for page $p_{i}$, $P R\left(p_{i}\right)$, is formulated as in Equation 7 where $\operatorname{In}\left(p_{i}\right)$ and $\operatorname{Out}\left(p_{j}\right)$ are the total numbers of incoming and outgoing links for pages $p_{i}$ and $p_{j}$ respectively, $\mathrm{N}$ represents the total number of pages, and $\lambda$ the probability that an internet surfer will continue navigating to other pages 
randomly, known as a damping factor. The recommended value for $\lambda$ is 0.85 but can be set to any number between 0 and 1.

$$
P R\left(p_{i}\right)=\frac{1-\lambda}{N}+\lambda * \sum_{p_{j} \in \operatorname{In}\left(p_{i}\right)} \frac{\operatorname{PR}\left(p_{j}\right)}{\operatorname{Out}\left(p_{j}\right)}
$$

In the summarization context, we rank document sentences instead of web pages; hence sentences play the roles of webpages. For a document graph, intra-sentence semantic similarities take the place of incoming and outgoing links in the computation of sentence ranks. The rank of each sentence indicates its salience which depends on the number and the strength of semantic links connecting each sentence to the rest of document sentences. In other words, sentences with strong connections (high semantic similarities) are more likely to be candidates for summary inclusion than those with a lot of weak connections (low similarities).

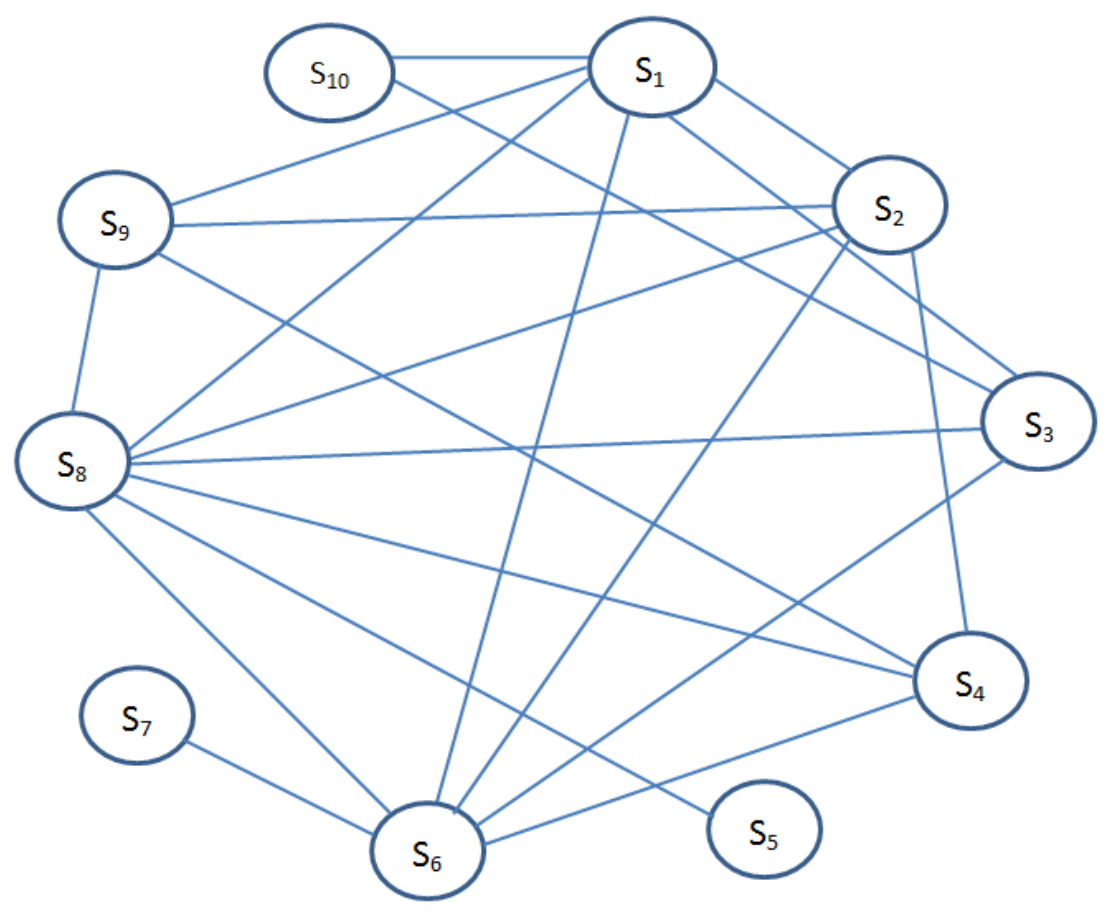

Figure 8: Sentence similarity graph of the example document in Figure 7 for clarity, the graph only shows links with weights $\geq 0.05$, while the full link values are given in Table 6

To examplify our reasoning, a short document of 10 sentences taken from the DUC2002 dataset is represented in Figure 7. To apply the proposed approach, the document is first pre-processed and parsed with SRL to allow the extraction of predicate verbs (semantic frames) and associated semantic role-sets. Figure 8 shows the sentence similarity graph of the document. Note that, for the purpose of legibility, self-links and intra-sentence links with similarity scores below 0.05 are omitted from Figure 8 . However, Table 6 lists the SRLESA based intra-sentence similarity scores of the entire document. In addition, the numbers in Table 7 represent the title-sentence similarities whose averages are combined with intrasentence similarities to form the edge weights in accord with equations (5 6). Finally, Table 8 
shows final sentence ranks in brackets after running the PageRank algorithm for 20 iterations, which is where the ranking algorithm empirically reached its steady state. The impact of the title-sentence similarity scores is evident on the sentence ranking where sentences with title similarities, namely 1, 2, 9 (see Table 7) are highest ranked as shown in Table 8, alluding to the importance of the sentences semantically connected with the document title.

From the given sentence ranking scores in Table 8 , the document sentences are ranked according to their importance as in the rank order row of the table with the most and least salient sentences being the first and the seventh respectively. For the purpose of summary generation, the highest ranked sentences of not more than the required summary length (100 words), as set by the DUC2002 guidelines, are selected as a summary. With this restriction in force, the extracted summary, which is given in Figure 9 comprises of sentences 1, 2, 9, and part of sentence 8 .

Table 6 Intra-sentence similarities of the example document in Figure 7 using the SRL-ESA based semantic similarity measure

\begin{tabular}{lllllllllll}
\hline Sent. No & 1 & 2 & 3 & 4 & 5 & 6 & 7 & 8 & 9 & 10 \\
\hline 1 & 1.0 & & & & & & & & & \\
2 & 0.1436 & 1.0 & & & & & & & & \\
3 & 0.0690 & 0.0458 & 1.0 & & & & & & & \\
4 & 0.0472 & 0.1320 & 0.0234 & 1.0 & & & & & & \\
5 & 0.0477 & 0.0210 & 0.0246 & 0.0217 & 1.0 & & & & & \\
6 & 0.1123 & 0.0789 & 0.1054 & 0.26 & 0.0044 & 1.0 & & & & \\
7 & 0.0023 & 0.0012 & 0.0137 & 0.0059 & 0.0033 & 0.0517 & 1.0 & & & \\
8 & 0.2399 & 0.0685 & 0.0654 & 0.2733 & 0.0683 & 0.1279 & 0.0071 & 1.0 & & \\
9 & 0.4692 & 0.2514 & 0.0407 & 0.3291 & 0.0212 & 0.0081 & 0.0 & 0.3513 & 1.0 & \\
10 & 0.00025 & 0.0002 & 0.1297 & 0.0001 & 0.0015 & 0.0 & 0.0 & 0.0015 & 0.0 & 1.0 \\
\hline
\end{tabular}

\begin{tabular}{lllllllllllll}
\hline Table 7 Sentence-title similarity scores of the example document in Figure 7 \\
\hline Sentence No & 1 & 2 & 3 & 4 & 5 & 6 & 7 & 8 & 9 & 10 \\
\hline Title similarity score & 0.3333 & 0.13094 & 0.0 & 0.0 & 0.0 & 0.0 & 0.0 & 0.0 & 0.3448 & 0.0 \\
\hline \multicolumn{10}{l}{} \\
\hline \multicolumn{10}{l}{ Table 8 Sentence-ranks after 20} & iterations of the example document in Figure 7 & \\
\hline Sentence No & 1 & 2 & 3 & 4 & 5 & 6 & 7 & 8 & 9 & 10 \\
\hline Sentence Ranks & 1.446 & 1.095 & 0.912 & 0.968 & 0.862 & 0.938 & 0.85 & 0.979 & 1.057 & 0.894 \\
Rank order & 1 & 2 & 7 & 5 & 9 & 6 & 10 & 4 & 3 & 8 \\
\hline
\end{tabular}

Ousted East German leader Erich Honecker will not stand trial in East Germany as long as the formerly Communist country exists, a West German newspaper reported. The Hamburg-based Bild am Sonntag said Saturday that it would report in its Sunday editions that Honecker could be prosecuted in a united Germany, however, for violation of property laws. Bild said that Erich Mielke, the ex-head of East Germany's former secret police, was also unlikely to go to court in East Germany. He is under investigation on allegations of abuse of power, corruption, harboring terrorists and issuing shoot-to-kill orders to prevent East Germans.

Figure 9: A 100-word summary extracted from the example document in Figure 7 


\section{Experiments}

\subsection{Evaluation}

For the purpose of testing and validation, we used 21 clusters of 160 documents from the DUC2002 dataset, namely; (D061j, D062j, D064j, D065j, D066j, D067f, D068f, D070f, D071f, D072f, D074b, D075b, D076b, D077b, D079a, D080a, D081a, D083a, D108g, D109h, D113h). These sets are semi-randomly selected mainly from the first half of DUC2002. Table 9 provides some statistics of the used dataset. Each document or cluster in DUC2002 dataset comes with a model summary of various lengths ranging from $10 \sim 400$ words, which are either created or extracted by human experts to serve as a reference summary. The overall experimental design of the proposed summarizer is shown in Figure 3 and detailed in Section 5. As for the quantitative evaluation of the system against baselines and related works, we employed the Recall Oriented Understudy for Gisting Evaluation (ROUGE) 42, which is the most widely used official evaluation tool in text summarization. As given in Equation (8), the ROUGE determines the quality of a system summary by comparing its text to an ideal human summary (aka as a model/reference summary) and computing a group of ROUGE measures including ROUGE-N, ROUGE-SU, and ROUGE-L.

Table 9: Statistical description of the used dataset

\begin{tabular}{|c|c|c|c|c|}
\hline Cluster & Document \# & Sentences \# & Word \# & Task \\
\hline D061j & 6 & 238 & 3933 & SDS \& MDS \\
\hline D062j & 5 & 158 & 2869 & SDS \& MDS \\
\hline D064j & 7 & 254 & 4398 & SDS \& MDS \\
\hline D065j & 8 & 371 & 5890 & SDS \& MDS \\
\hline D066j & 7 & 250 & 4127 & SDS \& MDS \\
\hline D067f & 6 & 168 & 2984 & SDS \& MDS \\
\hline D068f & 5 & 182 & 2791 & SDS \& MDS \\
\hline D070f & 11 & 250 & 3563 & SDS \& MDS \\
\hline D071f & 6 & 204 & 2380 & SDS \& MDS \\
\hline D072f & 13 & 483 & 8343 & SDS \& MDS \\
\hline D074b & 6 & 316 & 4057 & SDS \& MDS \\
\hline D075b & 10 & 328 & 6261 & SDS \& MDS \\
\hline D076b & 10 & 421 & 6765 & SDS \& MDS \\
\hline D077b & 10 & 429 & 6842 & SDS \& MDS \\
\hline D079a & 9 & 398 & 6873 & SDS \& MDS \\
\hline D080a & 11 & 653 & 10868 & SDS \& MDS \\
\hline D081a & 12 & 403 & 6604 & SDS \& MDS \\
\hline D083a & 6 & 276 & 4622 & SDS \& MDS \\
\hline D108g & 10 & 296 & 4081 & SDS \& MDS \\
\hline D109h & 10 & 275 & 4262 & SDS \& MDS \\
\hline D113h & 5 & 183 & 2833 & SDS \& MDS \\
\hline
\end{tabular}

Where $N$ is the length of the n-gram $\left(\operatorname{gram}_{n}\right), \operatorname{Count}\left(\operatorname{gram}_{n}\right)$ is the number of n-grams $\left(\operatorname{gram}_{n}\right)$ in the reference summary while $\operatorname{Count}_{\text {match }}\left(\operatorname{gram}_{n}\right)$ is the maximum number of 
n-grams co-occurring in the system summary and the collection of reference summaries (RefSumm). A gram $_{n}$ refers to a sequence of $\mathrm{n}$ words, for instance two-word is called bigram.

\subsection{Results and Discussion}

To extract a document representative summary, S, for SDS and MDS, we made use of the semantic graph interconnectivity among document sentences to calculate a quality ranking for each sentence. To this end, PageRank algorithm is iteratively run on the document similarity graphs (see Section 5.4) until it converges. All sentences are ranked equally at the beginning of the algorithm, which is run recursively on document similarity graphs until it reaches a steady state. Each sentence is ranked depending on the number of other connected sentences and the strength of the similarity between it and the rest of the document or cluster sentences. Sentences with high semantic similarity and linked with many other document sentences are favoured and ranked higher. These are then sorted according to their ranks and selected as a summary. In most cases, our experiments showed that the employed ranking algorithm converges before reaching the $20^{\text {th }}$ iteration.

Following NIST's ${ }^{5}$ dataset construction guidelines, the lengths of extracted summaries are 100 and 200 words for SDS and MDS respectively. Table 10 and Table 11 show the quality of the system summaries produced for SDS and MDS in terms of the average ROUGE recall scores of the selected measures. The choice of the measures is made on the basis of the findings in [42, where researchers reported that the measures used for Qf-MDS are the ones that work well for topic-focussed MDS and that the measures, ROUGE-N $(\mathrm{N}=1,2)$, ROUGE-L, and ROUGE-SU4 effectively reflect the effectiveness of generic SDS systems. The numbers in the square brackets following the ROUGE scores are minimum and maximum ROUGE recall values in the format [min-max]

Table 10: The overall results of the SRL-ESA graph-based single document summarisation (SDS): average recall of the four selected ROUGE measures at $95 \%$ confidence interval.

\begin{tabular}{lccc}
\hline Measure & Recall & Precision & F-measure \\
\hline ROUGE-1 & $0.504[0.228-0.79]$ & $0.431[0.212-0.665]$ & $0.462[0.232-0.676]$ \\
ROUGE-2 & $0.235[0.029-0.537]$ & $0.201[0.023-0.510]$ & $0.216[0.026-0.518]$ \\
ROUGE-L & $0.335[0.132-0.592]$ & $0.286[0.101-0.560]$ & $0.307[0.121-0.572]$ \\
ROUGE-SU4 & $0.254[0.062-0.534]$ & $0.216[0.061-0.487]$ & $0.232[0.063-0.496]$ \\
\hline
\end{tabular}

Table 11: The overall results of the SRL-ESA graph-based multi-document summarisation (MDS): average recall of the three selected ROUGE measures at $95 \%$ confidence interval.

\begin{tabular}{lccc}
\hline Measure & ROUGE-1 & ROUGE-2 & ROUGE-SU4 \\
\hline Recall & $0.474[0.336-0.642]$ & $0.212[0.068-0.380]$ & $0.246[0.106-0.413]$ \\
Precision & $0.427[0.318-0.529]$ & $0.190[0.064-0.323]$ & $0.220[0.100-0.336]$ \\
F-measure & $0.449[0.327-0.577]$ & $0.201[0.066-0.341]$ & $0.232[0.103-0.371]$ \\
\hline
\end{tabular}

\footnotetext{
${ }^{5}$ National Institute of Standards and Technology
} 


\subsubsection{Generalization and Data Size Effect}

To draw a generalization of the obtained results, we investigated the impact of data size on the performance of the summarisers. Tables 12 illustrate how changing data size, in terms of the number of documents for SDS (Table 12 ) and the number of document sets for the MDS (Table 13), affects the summariser's performance. Visualizations of the same results are also shown in Figure 10. Interestingly, we found that system results when tested on varying data sizes remain consistent on average, particularly for the single document summarization. However, experimental results seem to be less consistent for MDS as compared to the SDS task. This is shown in Table 13 where the scores of the ROUGE measures have larger deviations compared to the corresponding scores for the SDS task in Table 12 . Possibly, this is because of the large document sizes, in terms of the number of sentences after merging multidocument clusters. It could also be due to the high compression rate needed to summarize long merged cluster documents. Succinctly, this set of results indicate that the variation of the evaluation data size has little influence on the quality of the summaries particulary for the single-document summarization. Therefore, we may deduce that the proposed SRLESA graph-based SDS and MDS system is scalable, which leads us to generalize that the evaluation can represent a dataset of any size.

Table 12: Impact of data size (number of documents - NoD) for Single Document Summarization (SDS) - all figures are rounded to 3 significant figures.

\begin{tabular}{lccc}
\hline NoD & ROUGE- & ROUGE-2 & ROUGE-L \\
\hline 10 & 0.492 & 0.206 & 0.290 \\
20 & 0.475 & 0.211 & 0.300 \\
30 & 0.501 & 0.253 & 0.337 \\
40 & 0.503 & 0.247 & 0.334 \\
50 & 0.504 & 0.245 & 0.337 \\
60 & 0.495 & 0.228 & 0.321 \\
70 & 0.469 & 0.229 & 0.321 \\
80 & 0.492 & 0.225 & 0.319 \\
90 & 0.488 & 0.218 & 0.315 \\
100 & 0.494 & 0.226 & 0.324 \\
110 & 0.497 & 0.229 & 0.328 \\
120 & 0.504 & 0.235 & 0.335 \\
\hline
\end{tabular}

Table 13: Impact of data size (number of clusters - NoC) for Multi Document Summarization (MDS) - all figures are rounded to 3 significant figures.

\begin{tabular}{lccc}
\hline NoC & ROUGE-1 & ROUGE-2 & ROUGE-SU4 \\
\hline 1 & 0.471 & 0.197 & 0.229 \\
2 & 0.336 & 0.068 & 0.106 \\
3 & 0.417 & 0.137 & 0.183 \\
4 & 0.642 & 0.380 & 0.413 \\
5 & 0.587 & 0.359 & 0.370 \\
6 & 0.513 & 0.248 & 0.274 \\
7 & 0.430 & 0.105 & 0.158 \\
8 & 0.502 & 0.231 & 0.259 \\
9 & 0.469 & 0.282 & 0.305 \\
10 & 0.378 & 0.117 & 0.160 \\
\hline
\end{tabular}




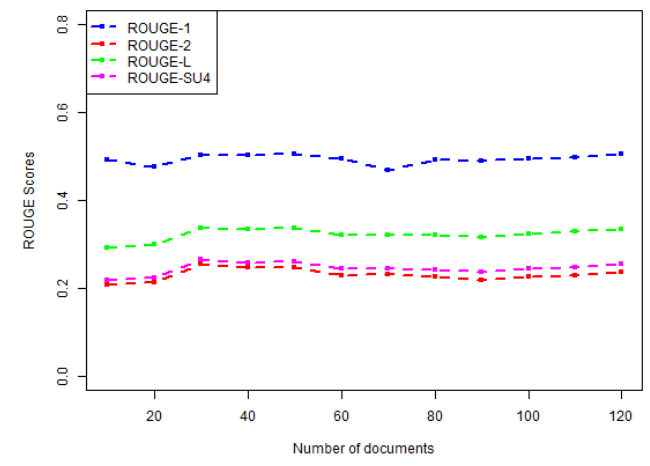

(a) (A) Impact of data size for SDS

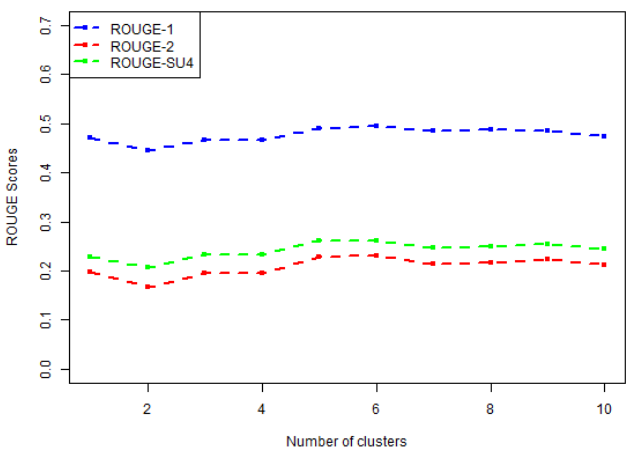

(b) (B) Impact of data size for MDS

Figure 10: Impact of data size on the SRL-ESA graph-based SDS (A) and MDS (B) tasks

A very commonly used statistical technique for generalization though is the concept of confidence intervals (CI). It is the range of values that is thought to include the true representative value, or the mean, of the entire results. In our case, that figure is the average ROUGE score of the entire data. Luckily, for our results, this generalization has been implemented in the evaluation metric, the ROUGE measure, which applies a bootstrap resampling technique to generalize evaluation results 42 . Specifically, it uses a $95 \%$ confidence interval, which indicates the range within which any result in the evaluation is true $95 \%$ of the time for the entire data.

\subsubsection{Comparison with Benchmark Methods and Related Works}

In the previous section, we have shown how changing the data size has little impact on the performance of the proposed summarizer, which allowed us to generalize system results. In this section, we compare our results with benchmark methods including Microsoft Word Summariser and related state-of-the-art systems. The selection of the aforementioned comparators was based on their relatedness to the current work in terms of the implementation and evaluation dataset. In the following paragraphs, we provide a brief introduction to each of the used comparators.

- Microsoft Word Summariser is a summarisation tool embedded in the Microsoft Word Application. This summarizer uses term frequencies to calculate the relative importance of each sentence in a document. It is widely used in related studies [43, 44, 45, 46, 47] as a benchmark method for automatic summarisation systems.

- System 19 is the best performing summarization system at the relevant competition in the Document Understanding Conference (DUC) [50]. The summarizer, which was implemented for the evaluation of the DUC 2002 dataset, can be be used to produce summaries from both single and multiple documents.

- Binwahlan et al. (2010) is a hybrid model for automatic single-document summarization combining diversity, swarm and fuzzy methods to achieve three criteria; avoiding redundancy in the generated summary, identification of the most appropriate text features for sentence scoring and the optimization \& adjustment of feature weights. 
- Wan (2010) is system which combines SDS and MDS tasks by examining the mutual influences between them. It proposes a unified graph model called CoRank which establishes word-sentence relationship. The proposed approach combines local saliency for the identification of sentence importance in a particular document with global saliency used to indicate the importance of a sentence in the whole document cluster.

- Abuobieda et al. (2013) is a summarization approach which combines opposition-based machine learning and differential evolution. The opposition-based learning is used to optimize the performance of Differential Evolution (DE), which itself is used to adjust the algorithm's initial population instead of relying on a random number generator.

- Alguliyev et al. (2013) is an optimization-based multi-document summarization model. It uses sentence-to-document collection, summary-to-document collection and sentence-tosentence relations to select salient sentences and reduce redundancy in the summary.

- Kumar et al. (2014) is a topic-focused multi-document summarization strategy based on cross document relations and fuzzy reasoning. The approach follows three phases; extracting news components in the documents using Wordnet thresasure, named entity recognition and Gazateer lists, establishing cross document relations to identify relevant sentences, and finally the application of fuzzy reasoning to assign final sentence scores.

- Sankarasubramaniam et al. (2014) is a graph-based summarization system leveraged with Wikipedia concepts. The study maps document sentences to Wikipedia concepts for the construction of a bipartite sentence-concept graphs. The summarizer then ranks sentences based on the ranking of corresponding concept nodes. It is noteworthy that this is one of the closest studies to our work in terms of the implementation, e.g., the use of concept graphs and Wikpedia as external knowledge.

- Oliveira et al. (2016) is a generic single and multi document summarizer based on eighteen of the most widely used sentence scoring techniques including TextRank, TF-IDF, graph-based similarity, named-entities, sentence centrality and word co-occurrence. The scoring methods are used to compute the sentence importance in a document/cluster. This comparative study found that the strategy of combining features can lead to improved results.

- Ren et al. (2016) is a redundancy-aware summarization system which considers the importance of the sentences and the redundancy in the summary simultaneously instead of modelling them as two separate processes. Particularly, this approach first evaluates the importance of each sentence and then selects sentences to generate a summary based on both the importance scores and redundancy among sentences.

- Sun \& Zhuge (2018) is a SDS approach which extracts semantic link network from documents using graph representations of different language granularities (words, sentences, paragraphs and sections) as nodes and semantic links between the nodes. In this method, is-part-of, similar-to and co-occurrence links are built among various nodes to form Semantic Link Network for modelling the basic semantic structure of a document.

- Alguliyev et al. (2018) is a two-stage sentence selection summarization model based on clustering and optimization techniques. The first stage discovers all topics in a text by clustering the sentences set using kmeans method. In the second stage, optimization is employed to model the selection of salient sentences from document clusters. 
Table 14: Comparison of our results with benchmark methods and related works for Single Document Summarization (SDS) - all figures are rounded to 3 significant figures.

\begin{tabular}{lccc}
\hline Summarizer & ROUGE-1 & ROUGE-2 & ROUGE-L \\
\hline This system & $\mathbf{0 . 5 0 4 ( 1 )}$ & $\mathbf{0 . 2 3 5}(\mathbf{1})$ & $0.335(3)$ \\
MS Word & $0.471(5)$ & $0.212(8)$ & $0.310(5)$ \\
System 19 & $0.459(8)$ & $0.233(2)$ & $0.313(4)$ \\
Wan (2010) & $0.485(3)$ & $0.215(7)$ & - \\
Binwahlan et al. (2010) & $0.436(10)$ & $0.197(10)$ & $0.401(2)$ \\
Abuobieda et al. (2013) & $0.445(9)$ & $0.224(5)$ & $\mathbf{0 . 4 0 7}(\mathbf{1})$ \\
Sankarasubramaniam et al. (2014) & $0.460(6)$ & $0.230(4)$ & - \\
Oliveira et al. (2016) & $0.477(4)$ & $0.223(6)$ & - \\
Sun \& Zhuge (2018) & $0.460(7)$ & $0.207(9)$ & - \\
Alguliyev et al. (2018) & $0.491(2)$ & $0.231(3)$ & - \\
\hline
\end{tabular}

Table 15: Comparison of our results with benchmark methods and related works for MultiDocument Summarization (SDS) - all figures are rounded to 3 significant figures.

\begin{tabular}{lccc}
\hline Summarizer & ROUGE-1 & ROUGE-2 & ROUGE-SU4 \\
\hline This system & $0.474(2)$ & $0.212(3)$ & $0.246(2)$ \\
MS Word & $0.451(4)$ & $0.198(4)$ & $0.242(4)$ \\
System 19 & $0.467(3)$ & $0.213(2)$ & $0.245(3)$ \\
Wan (2010) & $0.383(5)$ & $0.079(7)$ & - \\
Alguliyev et al. (2013) & $\mathbf{0 . 4 9 9 ( 1 )}$ & $\mathbf{0 . 2 5 5}(\mathbf{1})$ & $\mathbf{0 . 2 8 6}(\mathbf{1})$ \\
Kumar et al. (2014) & $0.332(8)$ & $0.128(5)$ & $0.100(5)$ \\
Oliveira et al. (2016) & $0.358(7)$ & $0.078(8)$ & - \\
Ren et al. (2016) & $0.378(6)$ & $0.0961(6)$ & - \\
\hline
\end{tabular}

Tables 14 and 15 include a comparison of our results against baselines and other related state-of-the-art works for SDS and MDS tasks respectively. The numbers in the parenthesis following the scores of the ROUGE measures represent the ranking of each system in the list. As shown, the proposed system ranks the top in the ROUGE-1 and ROUGE-2 measures for the SDS task, while ranking the second place in the ROUGE-1 and ROUGE-SU4 measures for the MDS task. It is also clear that the proposed system is placed third based on the other two measures, namely the ROUGE-L (SDS) and the ROUGE-2 (MDS). This indicates the competency of the proposed SRL-ESA Wikpedia graph-based summarisation where it excelled in terms of ROUGE scores as compared to the benchmark and related state-of-theart summarisation methods. However, it goes without saying that the proposed approach underperforms the work of Alguliyev et al. (2018) in the MDS task, as shown in Table 15 .

Overall, the system's experimental results underline the advantages of the proposed summarization approach and the use of crowd-sourced knowledge with sentence-level semantic parsing for single and multi document summarisation tasks. In other words, the findings highlight the importance of using semantic parsing and argument terms' concepts to compute intra-sentence similarities. It also opens the door to further exploration of the applicability of our SRL-ESA Wikipedia based similarity measure to other similarity underpinned NLP tasks including paraphrase identification, plagiarism detection, conversational agents, text 
classification, among others. In addition, establishing semantic links under sentence level (i.e. the multi-node representation of sentence vertices) could be used for other NLP tasks such as concept extraction where ranking argument concepts is more appropriate than ranking sentences. Similarly, the proposed summarization framework can be applied to other languages included in Wikipedia provided the availability of equivalent semantic analysis techniques for that language.

\section{Conclusion and Future Work}

In this paper we proposed a text summarization approach encompassing both singledocument and multi-document summarization using semantic role labelig and Wikipediabased explicit semantic analysis. The SRL is used for the semantic representation of document sentences while the ESA algorithm facilitates the interpretation of semantically parsed sentences to indexed Wikipedia concepts. Semantic roles are paired if they fill the same semantic position in a sentence. Argument texts pertaining to the shared semantic roles are then projected to a vector of corresponding Wikipedia concepts where the intra-sentence semantic relatedness is computed from such concept vectors. A graph-based SDS \& MDS is built on the basis of developed SRL-Wikipedia based similarity measure. The paper also presented an experimental evaluation of the proposed methodology on a standard publicly available dataset from the relevant DUC conference. The obtained results revealed considerable performance improvements in the summary quality illustrating the power of the role-based semantic representation and its mapping onto a human generated natural concepts encoded in Wikipedia. The findings also suggest that the other NLP tasks underpinned by semantic similarity functions can also be enhanced with this approach.

As a future work, we intend to apply the SRL-ESA Wikipedia-based method to a number of other summarization tasks including opinion, product/service review and guided summarisation. Our immediate interest is on the latter as guided summarization involves the retrieval of a summary response to an event described in a user question and is thought to be the best way of summarizing documents relating to topics of template-like categories, such as attacks, accidents and natural disasters, investigations, and health \& safety. Such topics contain highly predictable facts such as who did what when and where and interestingly SRL can be the best tool for answering such event-based questions.

\section{References}

\section{References}

[1] Gambhir, Mahak, and Vishal Gupta, Recent automatic text summarization techniques: a survey., Artificial Intelligence Review, 47.1, pp. 1-66, 2017.

[2] Nenkova, Ani, Sameer Maskey, and Yang Liu, Automatic summarization."Proceedings of the 49th Annual Meeting of the Association for Computational Linguistics, Tutorial Abstracts of ACL, Association for Computational Linguistics, 2011.

[3] Mohamed, Muhidin, Automatic text summarisation using linguistic knowledge-based semantics. PhD Thesis, University of Birmingham, 2016. 
[4] Mohamed, Muhidin, and M. Oussalah, Similarity-based Query-focused Multi-document Summarization using Crowdsourced and Manually-built Lexical-Semantic Resources, Proceedings of The 9th IEEE International Conference on Big Data Science and Engineering (IEEE BigDataSE-15), 2015.

[5] Jiang, Yuncheng, et al., Feature-based approaches to semantic similarity assessment of concepts using Wikipedia, Information Processing \& Management, 51.3, pp. 215-234, 2015.

[6] Saif, Abdulgabbar, et al, Semantic concept model using Wikipedia semantic features, Journal of Information Science, 44.4, pp. 526551, 2017

[7] P. Wang, J. Hu, H.-J. Zeng, and Z. Chen, Using Wikipedia knowledge to improve text classification, Knowledge and Information Systems, 19.3, pp. 265-281, 2009.

[8] H. P. Luhn, The automatic creation of literature abstracts, IBM Journal of research and development, 2.2, pp. 159-165, 1958.

[9] Khan, Atif, Naomie Salim, and Yogan Jaya Kumar, A framework for multi-document abstractive summarization based on semantic role labelling, Applied Soft Computing, 30, pp. 737-747, 2015.

[10] Jha, Nishant, and Anas Mahmoud., Using frame semantics for classifying and summarizing application store reviews, Empirical Software Engineering, pp. 1-34, 2018.

[11] Aksoy, Cem, et al., Semantic argument frequency-based multi-document summarization, 24th International Symposium on Computer and Information Sciences (ISCIS), IEEE, 2009 .

[12] Yan, Su, and Xiaojun Wan, SRRank: leveraging semantic roles for extractive multidocument summarization, IEEE/ACM Transactions on Audio, Speech and Language Processing (TASLP), 22.12, pp. 2048-2058, 2014.

[13] Zhou, Yunqing, et al., Applying wikipedia-based explicit semantic analysis for querybiased document summarization, International Conference on Intelligent Computing, Springer, Berlin, Heidelberg, 2010.

[14] Erkan, Gnes, and Dragomir R. Radev, LexRank: graph-based lexical centrality as salience in text summarization, Journal of Artificial Intelligence Research, 22, pp. 457-479, 2004.

[15] Mihalcea, Rada, and Paul Tarau, TextRank: Bringing order into texts, Association for Computational Linguistics, 2004.

[16] Brin, Sergey, and Lawrence Page, Reprint of: The anatomy of a large-scale hypertextual web search engine, Computer networks, 56.18, pp. 3825-3833, 2012.

[17] Otterbacher, Jahna, Gunes Erkan, and Dragomir R. Radev, Biased LexRank: Passage retrieval using random walks with question-based priors, Information Processing \& Management, 45.1, pp. 42-54, 2009.

[18] Canhasi, Ercan, and Igor Kononenko, Semantic role frames graph-based multidocument sumarization, Proceedings of SiKDD'11, 2011.

[19] Wei, Furu, et al., A document-sensitive graph model for multi-document summarization, Knowledge and information systems 22.2, pp. 245-259, 2010. 
[20] Wan, Xiaojun., Towards a unified approach to simultaneous single-document and multidocument summarizations, Proceedings of the 23rd international conference on computational linguistics. Association for Computational Linguistics, 2010.

[21] Canhasi, Ercan, and Igor Kononenko, Weighted archetypal analysis of the multi-element graph for query-focused multi-document summarization, Expert Systems with Applications, 41.2, pp. 535-543, 2014.

[22] Azadani, Mozhgan Nasr, Nasser Ghadiri, and Ensieh Davoodijam, Graph-based biomedical text summarization: An itemset mining and sentence clustering approach, Journal of biomedical informatics, 84, 2018.

[23] Abdi, Asad, Siti Mariyam Shamsuddin, and Ramiz M. Aliguliyev, QMOS: Querybased multi-documents opinion-oriented summarization., Information Processing \& Management, 54.2, pp. 318-338, 2018.

[24] Morales, Laura Plaza, Alberto Daz Esteban, and Pablo Gervs, Concept-graph based biomedical automatic summarization using ontologies, Proceedings of the 3rd Textgraphs Workshop on Graph-Based Algorithms for Natural Language Processing, Association for Computational Linguistics, 2008.

[25] Sankarasubramaniam, Yogesh, Krishnan Ramanathan, and Subhankar Ghosh, Text summarization using Wikipedia, Information Processing \& Management 50.3, pp. 443-461, 2014.

[26] H. Zhuge, Multi-Dimensional Summarization in Cyber-Physical Society, Morgan Kaufmann, 2016.

[27] X. Sun and H. Zhuge, Summarization of Scientific Paper through Reinforcement Ranking on Semantic Link Network, IEEE ACCESS, 2018.

[28] Lloret, Elena, and Manuel Palomar, Text summarisation in progress: a literature review, Artificial Intelligence Review, 37.1, pp. 1-41, 2012.

[29] Allan, Keith, Natural language semantics, 2001.

[30] Baker, Collin F., Charles J. Fillmore, and John B. Lowe, The berkeley framenet project, Proceedings of the 17th international conference on Computational linguistics, Association for Computational Linguistics, 1, pp. 86-90, 1998.

[31] Fillmore, Charles J., and Collin Baker, A frames approach to semantic analysis, The Oxford handbook of linguistic analysis, pp. 313-339, 2010.

[32] Palmer, Martha, Daniel Gildea, and Paul Kingsbury, The proposition bank: An annotated corpus of semantic roles, Computational linguistics, 31.1, pp. 71-106, 2005.

[33] Salim, Naomie, L. Suanmali, and M. S. Binwahlan, SRL-GSM: a hybrid approach based on semantic role labeling and general statistic method for text summarization, Journal of Applied Sciences, 10.3, pp 166-173, 2010.

[34] Osman, Ahmed Hamza, et al. "An improved plagiarism detection scheme based on semantic role labeling." Applied Soft Computing, 12.5, pp. 1493-1502, 2012. 
[35] Gildea, Daniel, and Daniel Jurafsky, Automatic labeling of semantic roles, Computational linguistics, 28.3, pp. 245-288, 2002.

[36] R. Collobert, J. Weston, L. Bottou, M. Karlen, K. Kavukcuoglu and P. Kuksa, Natural Language Processing (Almost) from Scratch, Journal of Machine Learning Research (JMLR), 12.Aug, 2011.

[37] E. Gabrilovich and S. Markovitch, Wikipedia-based semantic interpretation for natural language processing, Journal of Artificial Intelligence Research, 34, pp. 443-498, 2009.

[38] Mehta, Parth, and Prasenjit Majumder, Effective aggregation of various summarization techniques, Information Processing \& Management, 54.2, pp. 145-158, 2018.

[39] R. Baeza-Yates and B. Ribeiro-Neto, Modern information retrieval, ACM press New York, 463, 1999.

[40] E. Gabrilovich and S. Markovitch, Overcoming the brittleness bottleneck using Wikipedia: Enhancing text categorization with encyclopedic knowledge, AAAI, 6, pp. 1301-1306, 2006.

[41] O. Egozi, S. Markovitch, and E. Gabrilovich, Concept-based information retrieval using explicit semantic analysis, ACM Transactions on Information Systems (TOIS), 29.2, p. 8, 2011.

[42] Lin, Chin-Yew. ROUGE: A package for automatic evaluation of summaries,Text Summarization Branches Out: Proceedings of the ACL-04 Workshop, 2004.

[43] L. Suanmali, N. Salim and M.S. Binwahlan, SRL-GSM: A Hybrid Approach based on Semantic Role Labeling and General Statistic Method for Text Summarization. Journal of Applied Sciences, 10.3, pp. 166-173, 2010.

[44] Binwahlan, Mohammed Salem, Naomie Salim, and Ladda Suanmali, Fuzzy swarm diversity hybrid model for text summarization, Information processing \& management, 46.5, pp. 571-588, 2010.

[45] Barzilay, Regina, and Michael Elhadad, Using lexical chains for text summarization, Advances in automatic text summarization pp. 111-121, 1999.

[46] Abuobieda, Albaraa, et al., Opposition differential evolution based method for text summarization, Intelligent Information and Database Systems. Springer Berlin Heidelberg, pp. 487-496, 2013.

[47] Xu, Songhua, Hao Jiang, and Francis Lau, User-oriented document summarization through vision-based eye-tracking, Proceedings of the 14th international conference on Intelligent user interfaces, ACM, 2009.

[48] Binwahlan, Mohammed Salem, Naomie Salim, and Ladda Suanmali, Fuzzy Swarm Based Text Summarization, journal of Computer Science, 5.5, pp. 338 - 346, 2009.

[49] Kumar, Yogan Jaya, et al, Multi document summarization based on news components using fuzzy cross-document relations, Applied Soft Computing, 21, pp. 265-279, 2014.

[50] Harabagiu, Sanda M., and Finley Lacatusu, Generating single and multi-document summaries with gistexter, Document Understanding Conferences, 2002. 
[51] Ferreira, Rafael, et al., Assessing sentence scoring techniques for extractive text summarization, Expert systems with applications, 40.14, pp. 5755-5764, 2013

[52] Oliveira, Hilrio, et al., Assessing shallow sentence scoring techniques and combinations for single and multi-document summarization, Expert Systems with Applications, 65, pp. 68-86, 2016.

[53] Alguliyev, Rasim M., et al., COSUM: Text summarization based on clustering and optimization, Expert Systems, p. e12340, 2018.

[54] Alguliev, Rasim M., Ramiz M. Aliguliyev, and Nijat R. Isazade, Multiple documents summarization based on evolutionary optimization algorithm, Expert Systems with Applications, 40.5, pp. 1675-1689, 2013.

[55] Ren, Pengjie, et al., A redundancy-aware sentence regression framework for extractive summarization, Proceedings of COLING 2016, the 26th International Conference on Computational Linguistics, pp. 33-43, 2016.

[56] Carbonell, Jaime, and Jade Goldstein., The use of MMR, diversity-based reranking for reordering documents and producing summaries, Proceedings of the 21st annual international ACM SIGIR conference on Research and development in information retrieval, ACM, 1998.

[57] Ferreira, Rafael, et al., emphA multi-document summarization system based on statistics and linguistic treatment, Expert Systems with Applications, 41.13, pp. 5780-5787, 2014.

[58] Mosa, Mohamed Atef, Arshad Syed Anwar, and Alaa Hamouda, A survey of multiple types of text summarization with their satellite contents based on swarm intelligence optimization algorithms, Knowledge-Based Systems, 163, pp. 518-532, 2019.

[59] Bollegala, Danushka, Naoaki Okazaki, and Mitsuru Ishizuka, A bottom-up approach to sentence ordering for multi-document summarization, " Information processing \& management, 46.1, pp. 89-109, 2010.

[60] Ferreira, Rafael, et al., Assessing sentence similarity through lexical, syntactic and semantic analysis, Computer Speech \& Language, 39, pp. 1-28, 2016. 